\title{
Mechano-chemical Stabilization of Three-dimensional Carbon
}

\section{Nanotube Aggregates}

Ryota Koizumi ${ }^{1}$, Amelia H. C. Hart ${ }^{1}$, Gustavo Brunetto ${ }^{2}$, Sanjit Bhowmick ${ }^{3}$, Peter S. Owuor ${ }^{1}$, John T. Hamel ${ }^{1}$, Anieph X. Gentles ${ }^{1}$, Sehmus Ozden ${ }^{1}$, Jun Lou ${ }^{1}$, Robert Vajtai ${ }^{1}$, S. A. Syed Asif $^{3}$, Douglas S. Galvão ${ }^{2 *}$, C. S. Tiwary ${ }^{* 1}$, P. M. Ajayan ${ }^{* 1}$

${ }^{l}$ Materials Science and NanoEngineering Department, Rice University, Houston, Texas, USA-77005

${ }^{2}$ Applied Physics Department, State University of Campinas - UNICAMP, 13083-859 Campinas, SP - Brazil.

${ }^{3}$ Hysitron, Inc., 9625 West $76^{\text {th }}$ Street, Minneapolis, MN 55344, USA.

Here we report combined study of experiments and simulations to understand how chemical functional groups can mechanically stabilize aggregates of carbon nanotubes (CNTs). Ultralow density aggregates of chemically functionalized CNTs, in the form of macro-scale spheres made by freeze-drying method, show mechanical stabilization and near complete elastic recovery during deformation. Simulations of interacting functionalized carbon nanotube aggregates show better structural retention compared to non-functionalized CNTs under compression, suggesting that the atomic level interactions between functional groups on adjoining CNTs help maintain structural rigidity and elastic response during loading. Aggregates of non-functionalized CNTs collapses under similar loading conditions. The dynamic mechanical responses of CNT macrostructures and mechano-chemical stabilization are directly observed using in-situ deformation inside a scanning electron microscope.

Corresponding Authors: cst.iisc@gmail.com (C. S. Tiwary), galvao@ifi.unicamp.br (Douglas S. Galvão) ajayan@rice.edu (P. M. Ajayan), Tel: 713-348-5904, Fax: 713-348-5423 


\section{Introduction}

Three dimensional (3D) architecture materials via bottom-up approach assembly, is an interesting growing area of research due to the ability to tailor the properties of the building blocks [1]. Using such techniques, many of the shortcomings, e.g., shaping, structural integrality, defects and material waste, can easily be overcome, as well as such approach also supports the growing demand for multi-functional and intelligent materials [2]. The discovery of carbon nanotubes (CNTs) [3] and graphene [4,5], as one and two dimensional (1D and 2D) materials respectively, brought a new wave of research interest to design $3 \mathrm{D}$ structures from these building blocks and similar materials. In order to interconnect these nanomaterials to build a large scale millimeter size 3D structure, several processing techniques based on bottom up approaches using physical, chemical and electrical methods have been reported in the recent past [6-14]. Most of the synthesis gives rise to ultra-light weight porous materials with high surface area and enhanced functional properties, which are useful in the applications of oil absorption, energy absorbing, and gas storage. The mechanical responses of most of the porous 3D architectures are valuable in high energy absorption applications.

Recently, chemical functionalization of CNTs was reported to be useful in interconnecting and developing porous structures. But till date the mechanical advantage of such atomic scale functionalization of CNT has not be clearly understood. In the current work, we report an easily scalable method of synthesizing 3D foam into spheres with millimeter to micrometer scales made up entirely of functionalized CNTs exhibiting completely elastic behavior until a high strain. In-situ mechanical system attached to a high resolution SEM has been used to conduct quasi-static uniaxial compression on the individual building block and macroscopic sphere. The complete elastic behavior of these CNTs spheres is further explained with help of detailed molecular dynamics (MD) simulations. The simulation addressed the role of functional groups in such kind of behavior. 


\section{Experimental}

Multiwall CNT (MWNT) powder was functionalized to attach carboxylic acid functional groups (-COOH) by nitric acid for 3-4 days in oil bath at 70-80 ${ }^{\circ} \mathrm{C}$. Functionalized MWCNT (50 mg) was sonicated in DI water $(5 \mathrm{~mL})$ for 1 hour, glutaraldehyde solution $(35 \mu \mathrm{L})$, resorcinol (6 mg) and borax $(0.5 \mathrm{mg})$ was added. The mixed solution was sonicated further for 4 hours, then, the solution was dropped directly into liquid nitrogen to form CNTs spheres ( $\varphi$ $\approx 20 \mu \mathrm{m}$ to $3-4 \mathrm{~mm}$ ). Fig. 1a inset shows the procedure used to prepare the CNTs spheres (supplementary video SV1). The spheres were further freeze-dried for 3-4 days (as shown in Fig. 1a), using Millrock Technology freeze drier at approximately $-50^{\circ} \mathrm{C}$ and 40 mtorr. After freeze-drying, the density of the CNTs sphere is approximately $0.003 \pm 0.001 \mathrm{gm} / \mathrm{cc}$.

Scanning electron microscope (SEM) pictures were taken by FEI Quanta 400 ESEM, at scanning electricity of 15 to $20 \mathrm{kV}$; Transmission electron microscope (TEM) pictures were taken by JEOL 2100 Field Emission Gun TEM; X-ray photoelectron spectroscopy (XPS) data was examined by PHI Quantera XPS using Al K $\alpha$ x-ray tube; and X-ray diffraction (XRD) data was measured by Rigaku D/Max Ultima II Powder XRD using Cu Ka X-ray tube.

\section{Simulation Methodology}

Classical molecular dynamics simulations (MD) were performed using the CHARMM[15,16] force field, as available in the LAMMPS code [17]. We used $(10,10)$ CNTs tubes of $180 \AA$ length. The functionalized structures (CNTs with carboxyl acid group (-COOH)) were generated randomly inserting these groups into along the tube external layers. For the present study we considered tubes with a level of functionalization of about $20 \%$. All the parameters to describe the bonded (bond, angle and dihedral) and non-bonded (van der Waals and Coulomb) interactions were obtained using CGenFF [18]. The porous 3D structures were generated in a way trying to mimic the experimental conditions where the tubes interact each other and the structure has a high level of porosity. In all cases a canonical ensemble (NVT) 
was used with the system temperature been controlled by a thermostat and the target temperature was $10 \mathrm{~K}$. To indent the system an artificial sphere with $90 \AA$ of radius was used. The sphere had a repulsive harmonic potential with the force in each atom given by $\mathrm{Fi}=-\mathrm{K}(\mathrm{r}-$ $\mathrm{R})^{2}$, where $\mathrm{K}=20 \mathrm{kcal} / \mathrm{mol} / \AA^{3}, \mathrm{r}$ is the distance between the center of the sphere and the atom $\mathrm{i}$ and $\mathrm{R}$ is the radius of the sphere. The indentation process is carried out by moving the center of the sphere towards the CNT arrangements with a constant velocity of $0.4 \AA$ A $/$ ss.

\section{Results and Discussion}

First, the morphological properties of the CNTs were analyzed, as shown in Fig. 1. The diameters of the CNTs spheres were measured in the SEM and found to vary from 20 micron to $3 \mathrm{~mm}$ depending on size of nozzle (Fig. 1b). Images were taken from different sections of the CNT sphere and it was observed to have an isotropic morphology (Fig. 1b, c). The side view SEM image (65degree tilted condition) shows porous architecture. The inset (Fig. 1d) shows uniform size formation in same process (shown in suplemntry video SV1). The porous structure consists of interconnected sheets (Fig. 1e). The densities of the spheres are measured to be $0.003 \pm 0.001 \mathrm{gm} / \mathrm{cc}$. The same method performed for CNT without functionalization does not form any structure. The functionalized method adopted in this study proved efficient as the CNTs were observed forming a continuous porous [19] interconnected network structure (Fig. 1e) and depending on size of droplet different sizes can be synthesized. Bright field TEM images show the functionalized CNT forming an interconnected waved CNT network. High resolution transmission electron microscope (HRTEM) images further confirmed the multiwall functionalized CNTs are sheet of the CNT sheets, which forms the interconnected 3D network (Fig. 1f). The HRTEM image also shows that despite the functionalization, the CNTs maintained their structural integrity, (Fig. 1f) unlike the commonly used etching method where the etchant degrades the CNTs walls[20]. The diameters of the multi-walled nanotubes are $25 \pm 5 \mathrm{~nm}$ (Fig. 1g). The structural, vibrational and 
surface characteristics were analyzed using Raman, XRD and XPS (Fig. 2a-c). The intensity of the $\mathrm{D}$ band was lower than the $\mathrm{G}$ band, showing fewer imperfections of the CNTs. Further, both bands are very sharp indicating the presence of carbon $\mathrm{sp}^{2}$ hybridization in the CNTs forming the sphere[21]. The $\mathrm{sp}^{2}$ hybridization was further confirmed by XPS which shows C$\mathrm{C}(\sim 284.5 \mathrm{eV}), \mathrm{C}-\mathrm{OH}(\sim 286.6 \mathrm{eV})$ and $\mathrm{O}=\mathrm{C}-\mathrm{OH}(\sim 290.2 \mathrm{eV})$ bonding from the carbon peak, which depicts the existence of the carboxylic acid functional group (-COOH) at the CNT seed. The XRD spectrum of the CNT spheres demonstrates the typical carbon peak $\mathrm{C}(002)$ at $26^{\circ}$ associated with graphene [22] and another peak at around $C(100)$ at $42^{\circ}$. A controlled experiments using prestin CNTs was performed, but the CNT aggregates disintegrate during drop in the liquid nitrogen. Hence, it cannot form any shape for further mechanical testing. A detailed spectroscopy analysis (FTIR, Raman, XPS) and thermal analysis showing functinlization is discussed in supporting information S1-S4. The effect of freez-drying on self assembly of the nanomaterial is explained in our previous work [19].

The mechanical behavior of the spheres was tested by quasi-static uniaxial compression loading inside an SEM using an SEM PicoIndenter, xrPI85 (Hysitron, Inc., USA). As it can be seen in Fig. 3a, after loading and unloading the spheres immediately return to their original shape (supplementary Video 2). The load displacement curve of a $3 \mathrm{~mm}$ sphere shows a complete elastic recovery, after a deformation of $10 \%$ strain. The compression was performed more than 5 times and the sphere displayed remarkable resilience, i.e., the material ability to recover back to its original dimension (Fig. 3a). The load-displacement curves also exhibit a small hysteresis loop in cyclic loading which indicates complete dissipating energy during deformation. In order to quantify the stiffness and modulus value using the load-displacement curve the log plot is fitted with linear line which gives rise to a slope of $1.48 \pm 0.02(\sim 1.5)$ and intercept of $-5.457 \pm 0.02$ for a $\mathrm{R}^{2}$ of 0.988 . The spherical body mechanical response under compression between two flat rigid surfaces may be approximated as 
$\mathrm{F}=\left[\frac{4 \mathrm{R}^{1 / 2} E}{3 \mathrm{X} 2^{3 / 2}\left(1-v^{2}\right)}\right] h^{3 / 2}$

Where $\mathrm{F}$ is the applied force, $\mathrm{R}$ is the initial radius of the CNT sphere, $\mathrm{E}$ is the Young's modulus and $v$ is the Poisson's ratio of the sphere is zero and $\mathrm{h}$ is displacement. The elastic modulus of the sphere is found to be $4.2 \mathrm{KPa}$ and stiffness from the unloading curve to be 3.2 $\mathrm{N} / \mathrm{m}$. In order to obtain further local mechanical response of individual CNTs, the tip of the sphere consisting of inter-connected CNT sheets were compressed using a smaller tip (Fig. 3b). The loading-unloading curve shows complete recovery with no hysteresis, and clearly confirms complete elastic behavior of the individual CNT sheets. The different low loads compression $(1,10,15,20,30,50 \mu \mathrm{N})$ of individual sheets also shows complete elastic recovery. The low load compression at $10 \mu \mathrm{N}$, in which a free standing sheet is compressed, shows an elastic modulus of $102.5 \pm 0.5 \mathrm{GPa}$ and $95.9 \mathrm{kN} / \mathrm{m}$ stiffness. This unexpected behavior of CNT spheres has not been reported before. Mechanical properties of different size aggregates $(50-500 \mu \mathrm{m})$ are measured using in-situ test. The variation of elastic modulus values for these different size spheres are shown in Fig. 3c. As we reduce size of these spheres from 500 to $50 \mu \mathrm{m}$, the elastic modulus increases from 0.015 to $0.08 \mathrm{GPa}$. Smaller size aggregates have better interconnect to void ratio, which results in higher modulus. The stress vs. strain curve of single sphere under compression is shown in Fig. 3d. The sphere deforms till $10 \%$ strain $(12 \mathrm{kPa}$ stress) and then the slope changes, as we increase strain the stress increases without failure. The SEM image of the foam shows the CNTs as shown in Fig. 3e-f, retains its porosity and interconnects. In conventional foams, failure occurs due to a combination of factors in the core, e.g., cell wall-thickness, non-uniform cell wall-thickness and misalignment of cell wall[23]. Failure generally initiates at the weakest and thinnest wall thickness of the core wall, progresses rapidly to other parts of the foam, and finally the whole foam structure fails. The strength of the foam is therefore closely linked to the mechanical stability of individual CNTs. Our CNT spheres perform better because we eliminate the 
mismatch in thickness of the cell walls; hence the load is transferred to the whole structure minimizing the structural failure of the core. In most of structural applications the future challenge is to engineer materials with low density and high elastic modulus (North West in Ashby map, Fig. 3d). This newly developed CNT spheres in this study has potential to generate a new class of materials with ultra-light weight (density lower than natural materials and foam structures) and high modulus. The mechanical properties of the sphere can be further improved in future using reinforcements or can be used as template for forming 3D composite using nanomaterials.

We have carried out molecular dynamic (MD) simulations using classical force field (CHARMM)[15,16], in order to gain further insights on the how CNT functionalization affect the way that tubes interact and how these interactions are important in determining the 3D network structure stability (under compression and elastic recovery). Our structural models are composed of functionalized and pristine CNTs assembled into 3D porous structures (Fig. 4). This specific structural arrangement tries to mimic the main features of the experimental CNT networks. We considered CNT of similar geometries with tubes with (Fig. 4a) and without (Fig. 4b) the presence of functional groups. The presence of the charged groups ($\mathrm{OH})$ from the carboxylic acid functional group $(-\mathrm{COOH})$ can affect the way that the tubes interact and the whole system stability, due to H-bond network formation and dispersion forces (mainly van der Waals). In order to determine the relative importance of these aspects for the whole structural stability under compression/decompression, we have also carried out MD simulations where the explicit charge interactions are turned off. During the simulation of the indentation processes a sphere, which represents a repulsive harmonic force is moved against the 3D CNT structures. The snapshot sequences, in Fig. 4c-top panel, show different stages of the indentation process. At $\mathrm{t}=210 \mathrm{ps}$, the indentation achieves maximum compression and the CNT porous structure preserves its main structural features, in comparison to the initial configuration $(\mathrm{t}=0 \mathrm{ps})$. When the same indentation procedure is repeated for the tubes 
with no functional groups (Fig. 4c- bottom panel), the action of the indenter causes the tubes to completely rearrange themselves and in the final configuration $(\mathrm{t}=360 \mathrm{ps})$ all the tubes are bundled (compacted) and aligned, and the main porous feature of the initial structure (porous) no longer exists. When the functionalized tubes are subject to the indenter deformations, the presence and strong interactions of the functional groups creates an interlock-like mechanism which makes more difficult the CNT sliding, rotations and/or bending (supplementary Video SV3). Analyzing a small interface contact area between two CNTs (Fig. 4c-middle panel), we can observe that due to the compression the tubes and/or functional groups come into closer contact, increasing the filling of the structural voids of the porous networks. On the other side, when the functional groups are absent, the interlock-like mechanism does not occur and we can observe that the tubes can easily move against each other, resulting in the structural collapse of the porous network. As expected, the existence of an interlock-like mechanism makes the energy required to slide two functionalized tubes against each other to be higher in comparison to the case of non-functionalized tubes. In the case of functionalized CNTs, the external energy injected into the system (mainly elastic one) through the indenter compression is not enough to significantly alter the porous network geometry, thus the structures exhibit a resilient behavior to compression, with an almost perfect elastic recovery. However, in the case of pristine CNTs, this energy it is enough to induce rotations and/or translational tube movements, resulting in tube alignment and/or bundling (supplementary Video SV4). The whole process can be better visualized in the movies included in the supplementary materials. For the case presented here, the indenter injected energy into the functionalized CNT system is not enough to allow significant tube rotate, because of the interlock-like mechanism prevents them. Thus, this energy is temporarily stored in the system in the form of elastic one (e.g., bending the tubes). This energy can be dissipated through heat (heat bath through the thermostat, see methodology section). 
During the indentation of the functionalized tubes the van der Waals energy change by small amounts $(\sim 0.05 \mathrm{kcal} / \mathrm{mol} / \mathrm{atom})$, for the case where the partial charges were explicitly considered (Fig. 4d - red line), and almost no change were observed for functionalized CNT with no partial charges (Fig. 4d - green line). However, the van der Waals energy changes around $0.45 \mathrm{kcal} / \mathrm{mol} / \mathrm{atom}$ for the non-functionalized CNTs. When the tubes are in the aligned configuration they maximize the contact area, increasing the van der Waals interaction, which yields a more compact structure (Fig. 4d-blue line). Using molecular dynamic simulations, we can understand that the most important feature to create a stable porous structure made of CNTs is the presence of functional groups. An interlock-like mechanism, created by the interaction and arrangement of these groups among tubes into close contact, is an essential feature to provide structural stability for the porous networks when subjected to mechanical compression (supplementary Video SV5). When these groups are not present, the action of the external indenter can induce translational movements in the tubes, leading to a compact bundle, due to the maximization of the contact area, which result in a weaker structure. The current simulation is representative and the deformation behaviour changes with other parameter such as length, indenter diameter, number of walls or level of fucntionalization. The combination of different functionalization can result in unique mechanochemistry [24-25]. The apropriate combination of these molecular chemical species can be utilized to tune the mechanical properties of these spheres.

For structural and functional applications in aerospace, catalysis, packaging, energy and biomedical induustries, there a huge demand for interconnected carbon nanostructures. There are several bottom up approches reported to make these interconnects [26-28]. The mechanical properties of such 3D architecture depends on the interconnected geometry [29]. The simple and easily scalable method reported in current work results in ultralow density structure with an isotropic mechanical propeties in all directions. The graphene based 3D foams and their chemical interconnected are found to be promissing materials [30-32], but the 
isotropic mechanical properties and low bending modulus poses a great challenge, which can be further explored using the current method.

\section{Conclusion:}

In summary, 3D spheres have been developed from functionalized carbon nanotubes with excellent resilience never reported before. These desirable properties were achieved by utilizing a simple freeze-drying method. We show that the diameter of the CNTs spheres can be controlled based on application necessity, which allow them to be used in a variety of matrices. Further, molecular dynamics simulations show the densely entangled functionalized carbon nanotubes are able to keep their structure intact compared to non-functionalized ones when subjected to load. It is this entanglement which leads to the high elasticity and high energy absorption. The current method can synthesize different sizes of spheres depending on liquid nozzle sizes, which can be further improved in future using automated system and optimized sizes and functionalization of carbon nanotube. The ultralow density spherical 3D architecture can have a broad range of applications such as energy storage, gas absorption $\left(\mathrm{CO}_{2}\right.$, Hydrogen, methane etc.), ultra light packaging in electronics, and as a template in chemical catalysis etc.

\section{Acknowledgements}

Authors acknowledge the funding support from U.S. Department of Defense: U.S. Air Force Office of Scientific Research for the Project MURI:"Synthesis and Characterization of 3-D Carbon Nanotube Solid Networks"Award No. FA9550-12-1-0035. GB and DSG acknowledge support from Brazilian Agencies CAPES, CNPq and FAPESP and they thank the Center for Computational Engineering and Sciences at Unicamp for financial support through the FAPESP/CEPID Grant \# 2013/08293-7. 


\section{References}

1. M. Scarselli, P. Castrucci, F. D. Nicola, I. Cacciotti, F. Nanni, E. Gatto, M. Venanzi, M. D. Crescenzi, Applications of Three-Dimensional Carbon Nanotube Networks, Beilstein J. Nanotechnol. 6 (2015) 792-798.

2. R. G. Mendes, A. Bachmatiuk, B. Büchner, G. Cuniberti, M. H. Rümmeli, Carbon Nanostructures as Multi-Functional Drug Delivery Platforms, J. Mater. Chem. B 1 (2013) 401-428.

3. S. Iijima, T. Ichihashi, Single-Shell Carbon Nanotubes of 1-Nm Diameter, Nature 363 (1993) 603-605.

4. A. K. Geim, K. S. Novoselov, The Rise of Graphene, Nat. Mater. 6 (2007) 183-191.

5. K. S. Novoselov et al., Electric Field Effect in Atomically Thin Carbon Films, Science 306 (2004) 666-669.

6. J. Kong, A. M. Cassell, H. Dai, Chemical Vapor Deposition of Methane for SingleWalled Carbon Nanotubes, Chem. Phys. Lett. 292 (1998) 567-574.

7. D. P. Hashim, N. T. Narayanan, J. M. Romo-Herrera, D. A. Cullen, M. G. Hahm, P. Lezzi, J. R. Suttle, D. Kelkhoff, E. Muñoz-Sandoval, S. Ganguli et al., Covalently Bonded Three-Dimensional Carbon Nanotube Solids via Boron Induced Nanojunctions, Sci. Rep. 2 (2012) 1-8.

8. Z. Fan, J. Yan, L. Zhi, Q. Zhang, T. Wei, J. Feng, M. Zhang, W. Qian, F. A. Wei, Three-Dimensional Carbon Nanotube/Graphene Sandwich and its Application as Electrode in Supercapacitors, Adv. Mater. 22 (2010) 3723-3728.

9. C. Kang, R. Baskaran, J. Hwang, B.-C.Ku, W. Choi, Large Scale Patternable 3Dimensional Carbon Nanotube-Graphene Structure for Flexible Li-Ion Battery, Carbon 68 (2014) 493-500. 
10. J. Lin, C. Zhang, Z. Yan, Y. Zhu, Z. Peng, R. H. Hauge, D. Natelson, J. M. Tour, 3Dimensional Graphene Carbon Nanotube Carpet-Based Microsupercapacitors with High Electrochemical Performance, Nano Lett. 13 (2013) 72-78.

11. Y. Zhu, L. Li, C. Zhang, G. Casillas, Z. Sun, Z. Yan, G. Ruan, Z. Peng, A.-R. O. Raji, C. Kittrell et al., A Seamless Three-Dimensional Carbon Nanotube Graphene Hybrid Material, Nat. Comms. 3 (2012) 1225.

12. C. Altavilla, M. Sarno, P. Ciambelli, A. Senatore, V. Petrone, New 'Chimie Douce' Approach to the Synthesis of Hybrid Nanosheets of MoS2 on CNT and their AntiFriction and Anti-Wear Properties, Nanotechnology 24 (2013) 125601.

13. M. Mazloumi, S. Shadmehr, Y. Rangom, L. F. Nazar, X. S. Tang, Fabrication of Three-Dimensional Carbon Nanotube and Metal Oxide Hybrid Mesoporous Architectures, ACS Nano 7 (2013) 4281-4288.

14. W.-Y. Jang, S. Kyriakides, A. M. Kraynik, On the Compressive Strength of Open-Cell Metal Foams with Kelvin and Random Cell Structures, Int. J. Solids Struct. 47 (2010) 2872-2883.

15. S. V. Sambasivarao, The biomolecular simulation program, J. Chem. Inf. Model. 18 (2013) 1199-1216.

16. K. Vanommeslaeghe, A. D. MacKerell, Automation of the CHARMM general force field (CGenFF) I: Bond perception and atom typing, J. Chem. Inf. Model. 52 (2012) 3144-3154.

17. S. Plimpton, Fast Parallel Algorithms for Short-Range Molecular Dynamics, J. Comput. Chem. 117 (1995) 1-19.

18. C. Steffen, K. Thomas, U. Huniar, A. Hellweg, O. Rubner, A. Schroer, TmoleX-A Graphical User Interface for TURBOMOLE, J. Comput. Chem. 31 (2010) 2967-2970.

19. P. M. Sudeep, T. N. Narayanan, A. Ganesan, M. M. Shaijumon, H. Yang, S. Ozden, P. K. Patra, M. Pasquali, R. Vajtai, S. Ganguli et al., Covalently Interconnected ThreeDimensional Graphene Oxide Solids, ACS Nano 7 (2013) 7034-7040.

20. X. Zhang, K. K. Yeung, Z. Gao, J. Li, H. Sun, H. Xu, K. Zhang, M. Zhang, Z. Chen, M. M. Yuen et al., Exceptional Thermal Interface Properties of a Three-Dimensional Graphene Foam, Carbon 66 (2014) 201-209.

21. L. Camilli, C. Pisani, E. Gautron, M. Scarselli, P. Castrucci, F. D’Orazio, M.

Passacantando, D. Moscone, M. D. Crescenzi, A Three-Dimensional Carbon Nanotube Network for Water Treatment, Nanotechnology 25 (2014) 065701. 
22. W.-L. Song, X.-T. Guan, L.-Z. Fan, W.-Q. Cao, C.-Y. Wang, M.-S. Cao, Tuning Three-Dimensional Textures with Graphene Aerogels for Ultra-Light Flexible Graphene/Texture Composites of Effective Electromagnetic Shielding, Carbon 93 (2015) 151-160.

23. D. Mouhamadou, T. Roland, P. Kekicheff, C. G. Analysis of the mechanical behavior of a polymer foam from 3D full-field strain measurement coupled with finite element modeling at the micro-structural scale, in 21ème Congrès Français de Mécanique (2013) $1-6$.

24. M. A. Kabbani, C. S. Tiwary, P. A. S. Autreto, G. Brunetto, A. Som, K. R. Krishnadas, S. Ozden, K. P. Hackenberg, Y. Gong, D. S. Galvao, R. Vajtai, A. T. Kabbani, T. Pradeep, P. M. Ajayan, Ambient solid-state mechano-chemical reactions between functionalized carbon nanotubes, Nature communications 6 (2015) 1-5.

25. M. A. Kabbani, C. S. Tiwary, A. Som, K. R. Krishnadas, P. A. S. Autreto, S. Ozden, K. Keyshar, K. Hackenberg, A. C. Chipara, D. S. Galvao, R. Vajtai, A. T. Kabbani, T. Pradeep, P. M. Ajayan, A generic approach for mechano-chemical reactions between carbonnanotubes of different functionalities, Carbon 104 (2016) 196-202.

26. S. Ozden, C. S. Tiwary, A. H. C. Hart, A. C. Chipara, R. R. Aburto, M. T. F. Rodrigues, J. T. Tijerina, R. Vajtai, P. M. Ajayan, Density Variant Carbon Nanotube Interconnected Solids, Adv. Mater. 27 (11) (2015) 1842-1850.

27. S. Ozden, G. Brunetto, N. S. Karthiselva, D. S. Galvão, A. Roy, S. R. Bakshi, C. S. Tiwary, P. M. Ajayan, Controlled 3D Carbon Nanotube Structures by Plasma Welding, Advanced Materials Interfaces (2016) 1500755.

28. S. Ozden, T. N. Narayanan, C. S. Tiwary, P. Dong, A. H. C. Hart, R. Vajtai, P. M. Ajayan, 3D Macroporous Solids from Chemically Cross-linked Carbon Nanotubes, Small 11(6) (2015) 688-693.

29. S. Ozden, Y. Yang, C. S. Tiwary, S. Bhowmick, S. Asif, E. S. Penev, B. I. Yakobson, P. M. Ajayan, Indentation Tests Reveal Geometry Regulated Stiffening of Nanotube Junctions, Nano letters 16 (1) (2015) 232-236.

30. S. Vinod, C. S. Tiwary, L. D. Machado, S. Ozden, J. Cho, P. Shaw, R. Vajtai, D. S. Galvão, P. M. Ajayan, Strain rate dependent shear plasticity in graphite oxide, Nano letters 16 (2) (2016) 1127-1131.

31. S. Vinod, C. S. Tiwary, P. A. S. Autreto, J. T. Tijerina, S. Ozden, A. C. Chipara, R.Vajtai, D. S. Galvao, T. N. Narayanan, P. M. Ajayan, Low-density three-dimensional foam using self-reinforced hybrid two-dimensional atomic layers, Nature Communications 5 (2014) 1-9.

32. D. Chakravarty, C. S. Tiwary, L. D. Machado, G. Brunetto, S. Vinod, R. M. Yadav, D. S. Galvao, S. V. Joshi, G. Sundararajan, P. M. Ajayan, Zirconia Nanoparticle 
Reinforced Morphology Engineered Graphene Based Foams, Adv. Mater. 27 (31) (2015) 4534-4543.

\section{Figure}




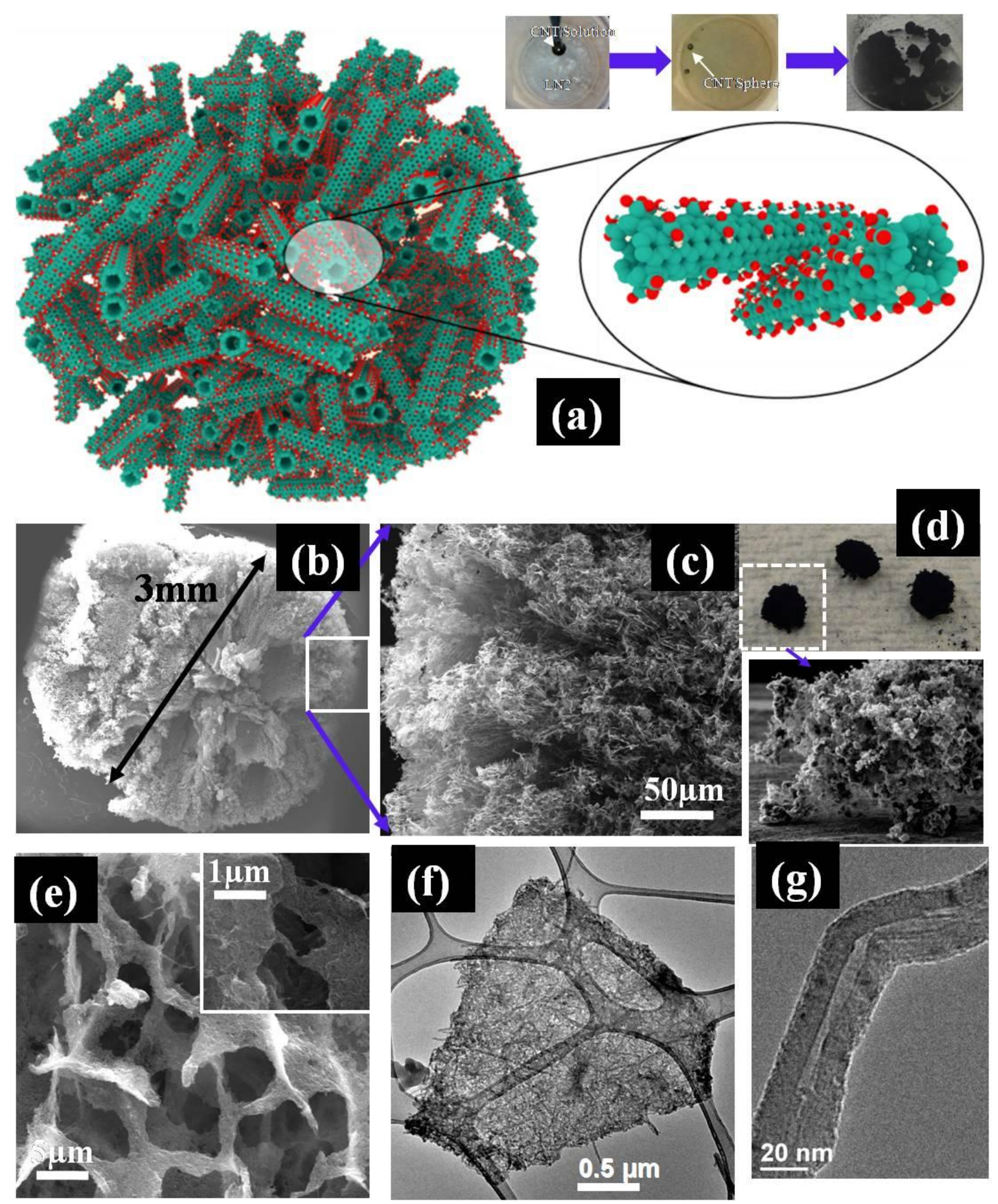

Fig 1. Morphological properties of CNTs spheres. (A) Schematic of the 3D CNTs spheres, inset shows fabrication method. (B) Nominal diameter of the CNTs spheres $(\sim 3 \mathrm{~mm})$. (C) High magnification SEM image of sphere showing porous morphology. (D) SEM image (using 65degree tilted) of side view of 3D architecture, Inset shows uniform size of the sphere (E) Highly porous interconnected network structure. Inset shows high magnification image of interconnect (F) Bright field TEM showing the CNTs sheets which form the interconnected 3D network. G) HRTEM images showing the structure integrity of multiwall CNTs spheres after functionalization. 

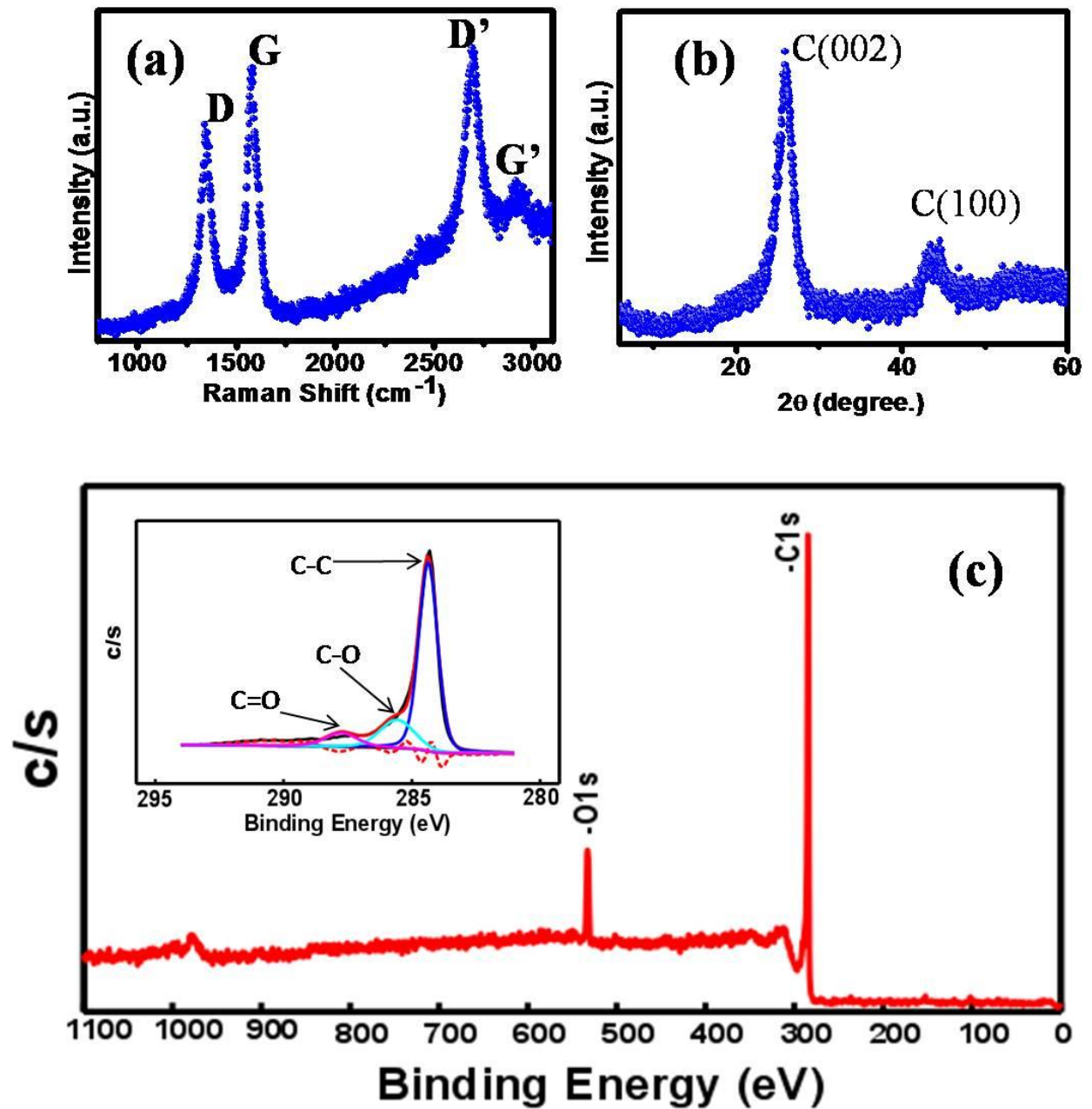

Fig 2. Surface characterization of CNTs spheres. (A) Raman spectrum showing lower D band intensity compared to G band. (B) XRD spectrum of the CNTs spheres demonstrates the typical carbon peak $\mathrm{C}(002)$ at $26^{\circ}$ associated with graphene and another peak at around $\mathrm{C}$ (100) at $42^{\circ}$. (C) XPS showing C-C ( 284.5 eV), C-OH ( 286.6 eV) and O=C-OH $(\sim 290.2$ $\mathrm{eV})$ bonding from the carbon peak, depicting the existence of the carboxylic acid functional group (-COOH) on CNT seed. 

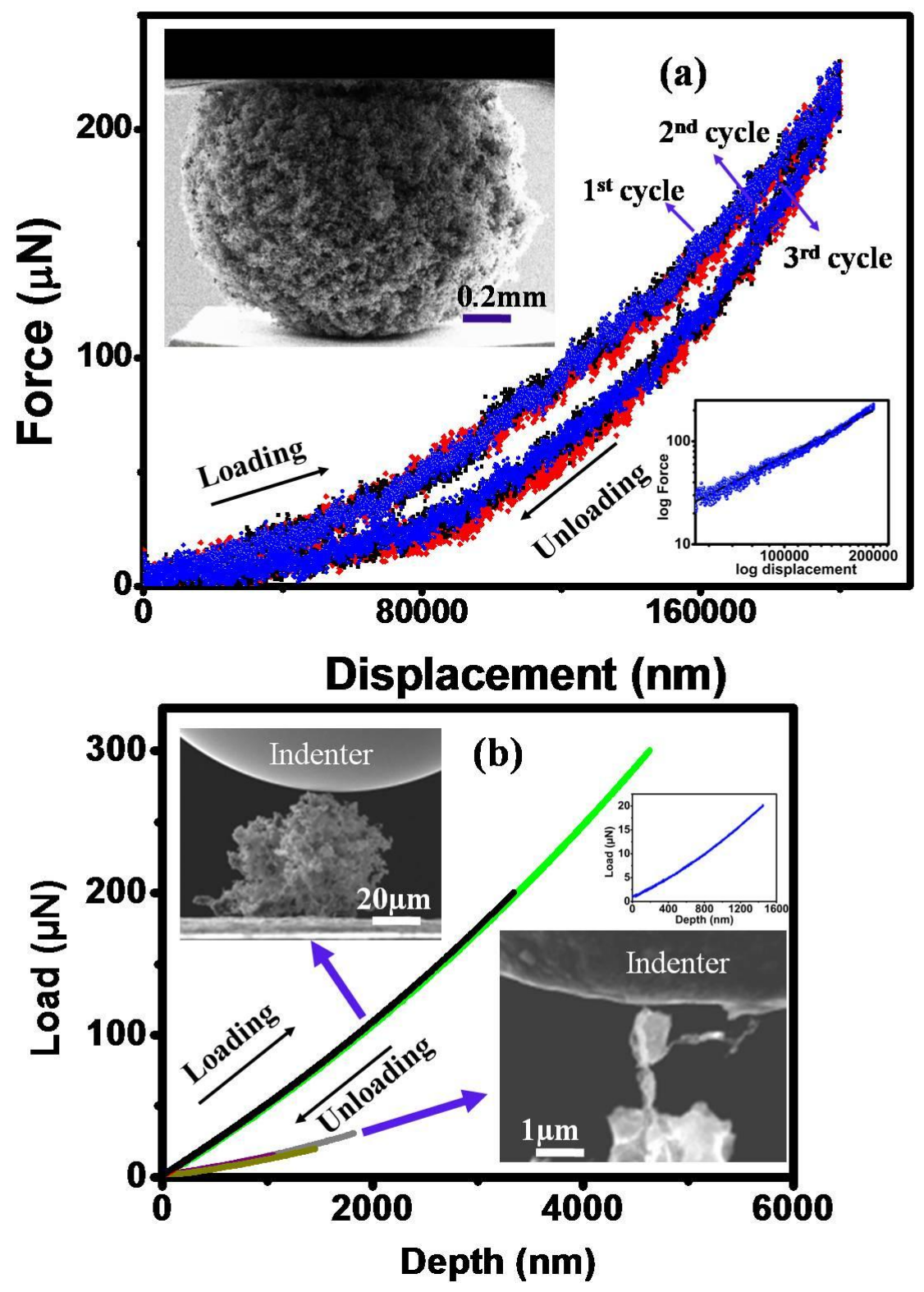

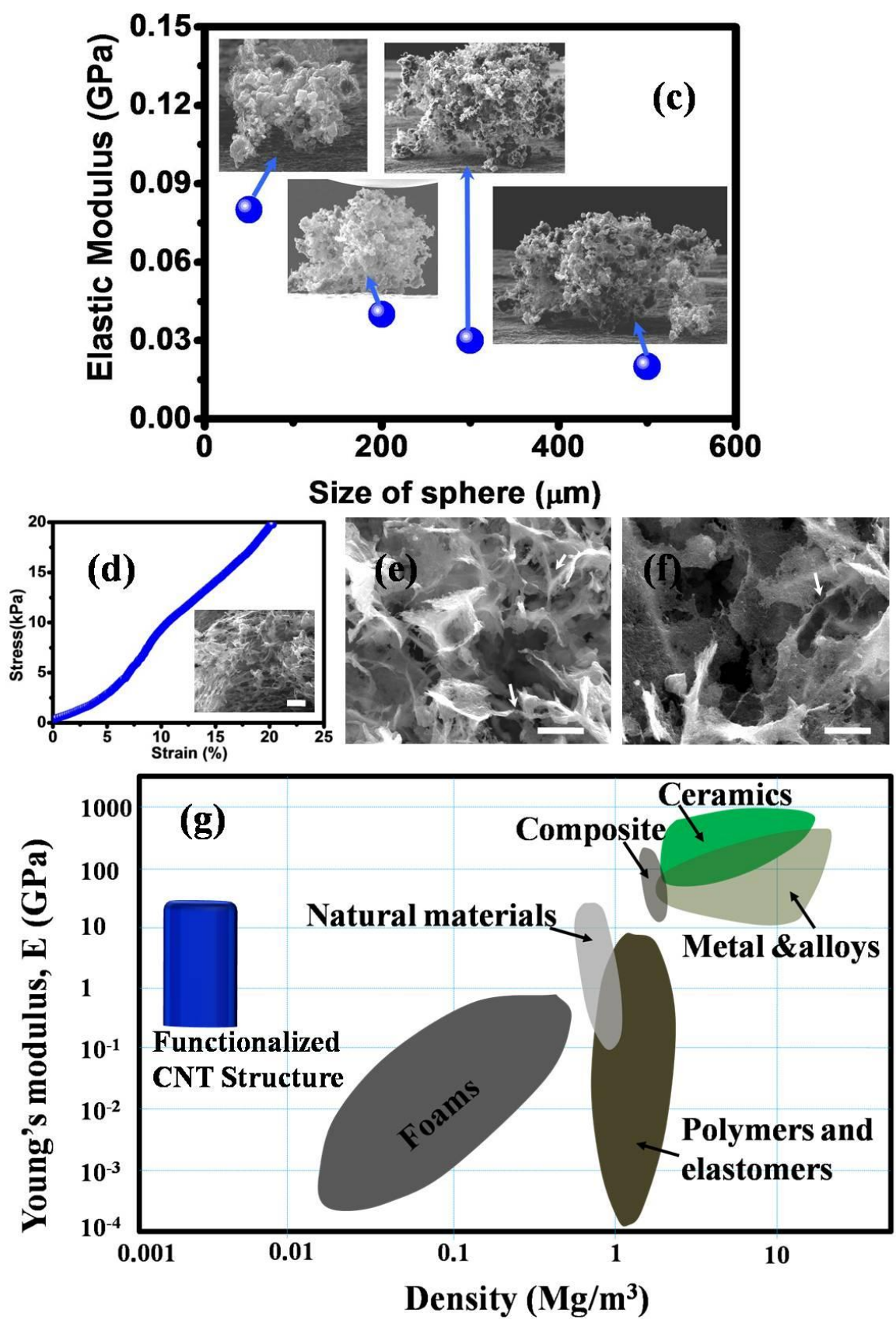

Fig 3. Mechanical properties of CNTs spheres. (A) Load vs. displacement curves for different cycle showing minimal hysteresis of the $3 \mathrm{~mm}$ CNTs spheres. It shows a complete elastic behavior (completely recovery) after a deformation of $10 \%$ strain. SEM showing loading condition of the sphere (left top), right bottom shows logarithmic plot of load and displacement. (B) High sensible load and displacement indenter setup (PI 85) inside SEM, the load displacement curve of individual sheets (low load) and few aggregates (higher loading). Inset shows (left top) SEM images during indentation test, inset (right bottom), Load vs. displacement curve for low load individual sheet (right top). (C) Variation of elastic modulus (in GPa) for different size CNTs aggregates. (D) Stress-strain curve of $3 \mathrm{~mm}$ sphere (E)-(F) Low and high magnification image of the compressed sample. (G) The modulus vs. density Ashby map for different materials with comparison of CNTs spheres. 
(a)
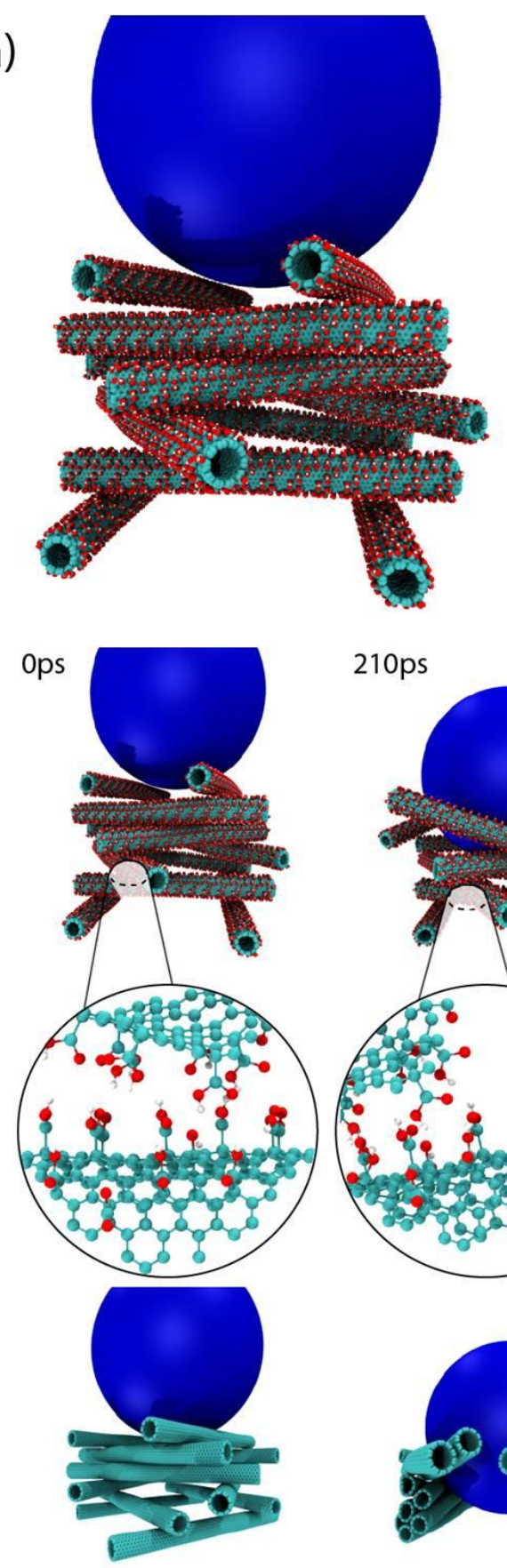

(b)

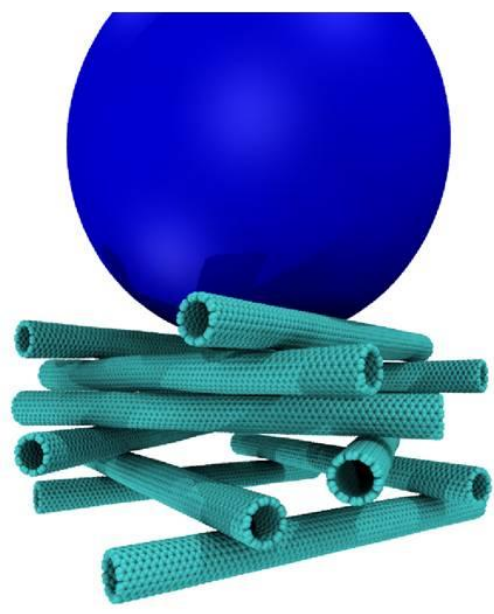

360ps

(c)
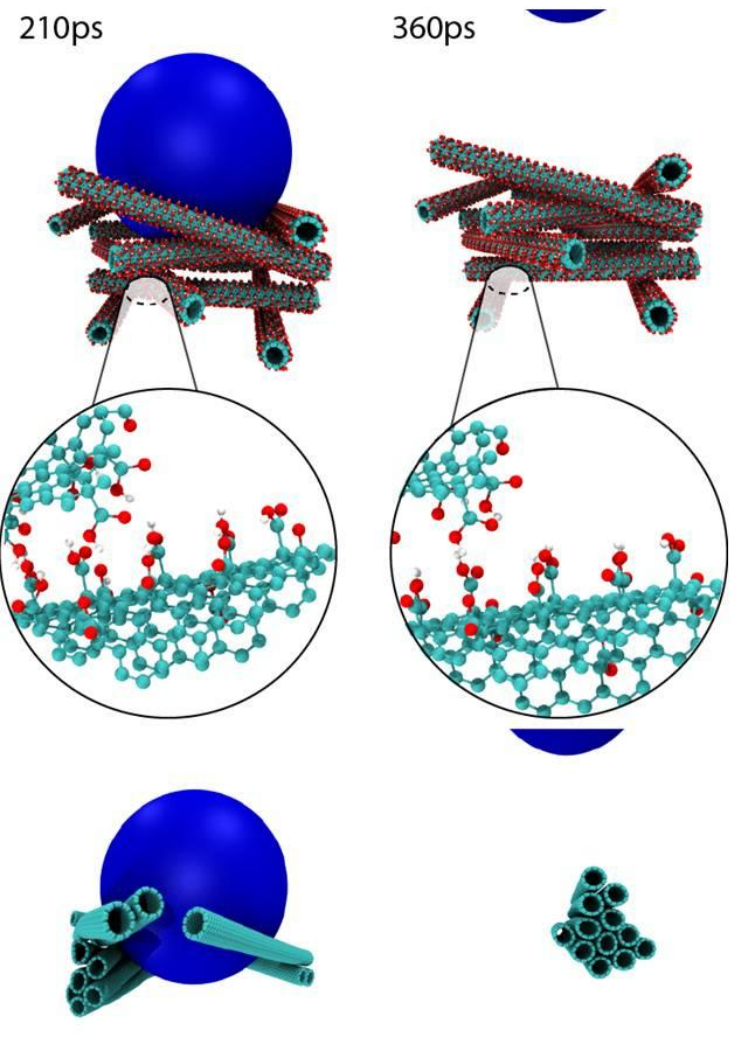


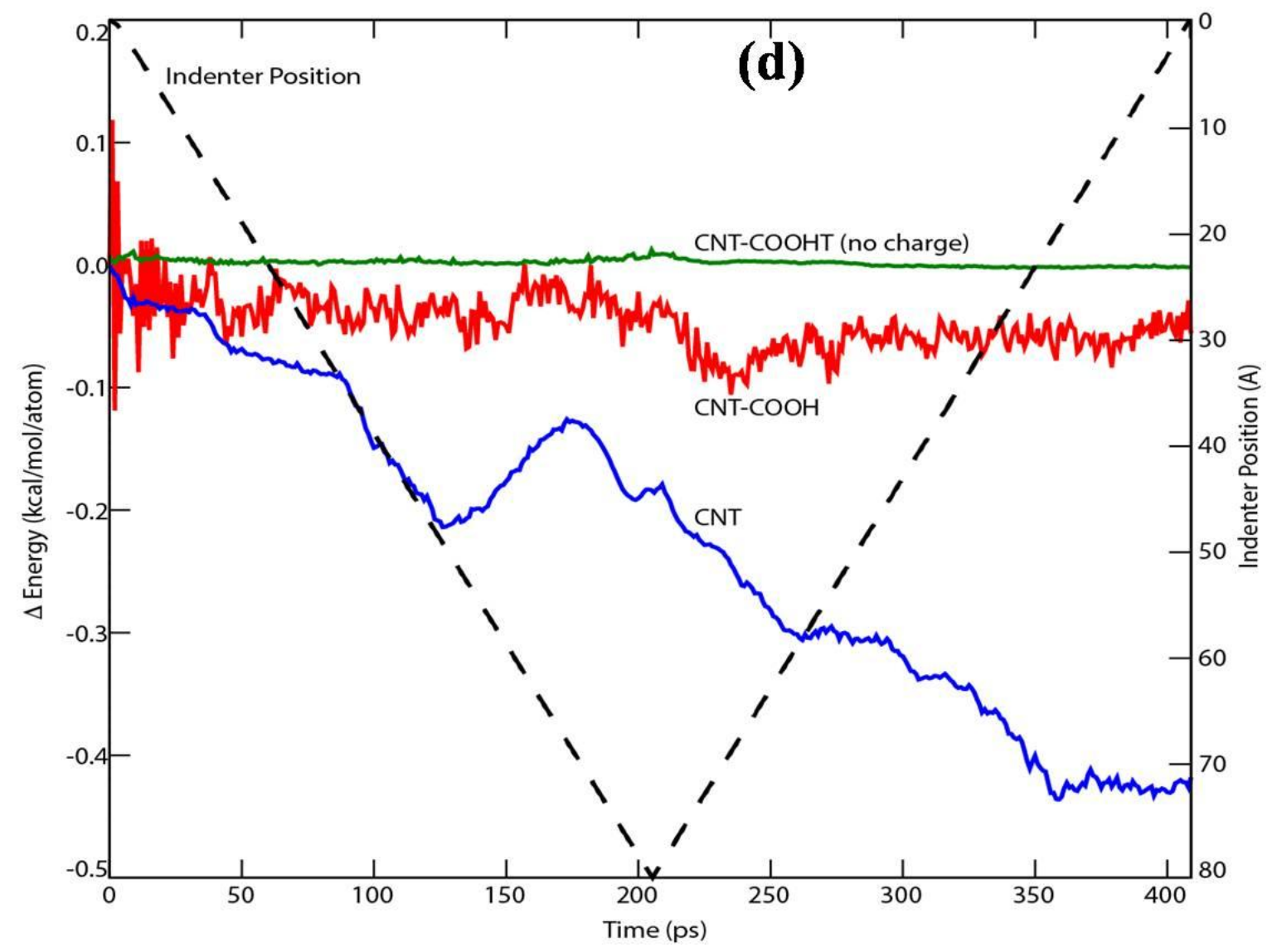

Fig 4. Molecular dynamic simulation (MD). (A) CNT-COOH assembled in a way to create a porous structure. (B) Similar assemble made of CNT. The blue sphere represents the external indenter used to compress locally the system. (C) Indentation process for functionalized and non-functionalized CNTs. (Top panel) time evolution of functionalized CNT under the action of an external indenter (blue sphere). At the maximum compression $(\mathrm{t}=210 \mathrm{ps})$ the porous structure is kept. When the indenter is completely removed $(\mathrm{t}=360 \mathrm{ps})$ no significant changes in the CNT porous network. (Middle panel) zoomed vision of a contact area between neighbor functionalized CNTs, creating an interlock mechanism. (Bottom panel) Indentation of a non-functionalized CNT showing a drastic change in the geometry due to the indenter action ( $\mathrm{t}=210 \mathrm{ps}$ ). (D) Van der Waals energy evolution during the indentation for the considered cases: (red) CNT functionalized with - $\mathrm{COOH}$ and partial charges considered, (green) CNT functionalized with - $\mathrm{COOH}$ but no partial charges and (blue) non-functionalized CNT. The dashed line shows the sphere indenter center (right axis). 